\title{
Editorial: Special issue on modelling and applications of computational Peer-to-Peer networks: Volume 2, Issue 1.
}

\author{
Nick Antonopoulos
}

Received: 14 November 2008 / Accepted: 19 November 2008/Published online: 18 December 2008

(C) Springer Science + Business Media, LLC 2008

Keywords Computational p2p networks

Peer-to-Peer Networks first emerged in the late 90's as specialised systems \& protocols to support distributed file sharing. Since then, P2P networking has gradually changed into a system methodology suitable for developing scalable resource discovery and sharing applications. A resource in this context is quite generic and encapsulates not just files but applications, services and computational resources such as storage and processing cycles.

So far the main emphasis of $\mathrm{P} 2 \mathrm{P}$ research has been on the performance optimisation of the large scale file discovery and sharing process. However, this work is not directly applicable to consumable resources such as services and processing cycles because these resources have two main characteristics: Firstly they are non-replicable and secondly (because they are consumable) they possess a highly volatile and unpredictable availability. The combined effect of the above two attributes is that distributed hash tables and numerous successful informed search techniques cannot directly be applied to this type of resources.

Computational P2P Networks are defined as scalable P2P systems designed to provide efficient discovery and access to non-replicable consumable resources as opposed to more traditional file-oriented P2P Networks. There is a growing demand for such systems as Internet Service Providers (ISPs) and content providers are increasingly looking towards exploiting the $\mathrm{P} 2 \mathrm{P}$ scalability and performance gains over traditional client-server architectures for efficiently

N. Antonopoulos $(\bowtie)$

Department of Computing, University Of Surrey,

Guildford, Surrey GU2 7XH, UK

e-mail: N.Antonopoulos@surrey.ac.uk distributing massive volumes of media content and services worldwide. This demand is further illustrated by the growing trend of using the Internet to access and use services such as processor farms, online storage facilities and commercial software applications. In all such scenarios, the underlying P2P infrastructures will have to cope with potentially massively varying demand for real time access to consumable resources from processing cycles to media streams.

This special issue aims to address the need for more research into the modelling of computational P2P Networks and their applications into the process of discovery and access to non-replicable resources. It includes six high quality papers selected after peer review from more than 20 submissions.

P2P Networks are increasingly being used as a middleware for the scalable discovery of services in Grid environments. In the paper, A Grouped P2P Network for Scalable Grid Information Service, Sahota et al., present PIndex; a grouped P2P Network that is used to provide scalable service discovery in Grids. It builds on the Globus architecture and dynamically splits the Grid information search space into manageable peer groups. Through modelling with Petri Nets and simulations the authors demonstrate that PIndex is resilient and can cope with large numbers of peer nodes.

The issue of security in P2P networking is gradually becoming one the main factors to determine the eventual success and adoption of this technology. It is of higher significance to networks providing access to services and consumable resources as opposed to replicable files. In the paper, Reputation based Friend-to-Friend Networks, Loukos \& Karatza describe an extension to anonymous networks for connecting to other nodes based on their reputation. Through the use of trust management techniques, peers can dynamically adjust the reputation values and use these to connect to honest nodes. Through extensive simulations the authors 
show that their mechanism can successfully identify malicious nodes in networks of different sizes.

Successful performance management of P2P Networks typically requires a good knowledge of network attributes such as number of nodes and interconnection degree. The task of acquiring accurate approximates of these values is non-trivial given the usual network churn and dynamic conditions inherently present in large scale P2P Networks. In the paper, Peer Sampling with Improved Accuracy, Ogston \& Jarvis present a new protocol, Eddy, which aims to provide accurate sampling of a P2P Network by minimising temporal and spatial dependencies between samples. Eddy maintains a balanced distribution of active system nodes in the presence of significant node churn. Appropriate simulations show that the protocol estimates the size of a P2P Network more accurately compared to a given benchmark.

A significant number of $\mathrm{P} 2 \mathrm{P}$ architectures rely on a randomlike initial interconnection of their nodes. This initial setup is usually far from optimal in the context of resource discovery and therefore the network needs to adapt its topology in order to improve the query efficiency in terms of latency and data traffic. In the paper, Adaptive Neighbourhood Selection in Peer-to-Peer Networks based on Content Similarity and Reputation, Pogkas et al., present the Adaptive Gnutella Protocol (AGP) which aims to improve the query efficiency and quality of search results in P2P Networks. The protocol achieves this by clustering nodes based on their content similarity and reputation. Free-riders and malicious nodes are in this way isolated and their impact on the network operation is significantly reduced. Through a series of simulations the authors demonstrate that the AGP improves the aforementioned metrics in various network settings.

A typical application of computational networks is to support real-time, large-scale multimedia stream processing. Providing Quality of Service (QoS) support in such environments is challenging due to the underlying heterogeneity of the network and the versatility of QoS demands. In the paper, Adaptive Component Composition and Load Balancing for Distributed Stream Processing Applications, Kalogeraki et al., present an adaptive component composition and load balancing algorithm that can cope with the aforementioned conditions. The algorithm enables the dynamic composition of multiple streams while ensuring that the load is fairly distributed on the relevant resources. It also adapts to any changes in the QoS requirements of the underlying applications. A set of combined simulations and prototype-based experiments illustrate the benefits of the algorithm in terms of performance and scalability.

Following on in the same theme, peer-to-peer IPTV applications are increasingly been considered as a serious candidate to online broadcasting. In particular, Joost is one of the latest such systems offering video-on-demand and realtime services. Measuring and evaluating this system in terms of traffic is useful and timely as this can provide us with important data on potential limitations of current state-of-theart IPTV systems. In the paper, Characterization of Signalling and Traffic in Joost, Alhaisoni \& Liotta study the impact of Joost on the underlying computer network through a careful investigation and analysis of the Joost traffic for both Videoon-Demand and real-time services. Through the application of appropriate statistical methods they reveal key strengths and shortcomings of current IPTV systems and yield a set of recommendations for the improvement of such systems.

In closing, I would like to thank all the authors who submitted their work to this special issue as well as the reviewers who, through their expert and insightful comments, helped improve significantly the quality of the submitted material. I would also like to thank the Editor-in Chief Prof Sherman Shen, the Associate Editor Dr Heather Yu and the editorial assistants Valerie Schofield and Christina Chua for their continued guidance and professional support throughout all the phases of this publication. I hope you will find the material in this first special issue of the Peer-to-Peer Networking and Applications interesting and useful.

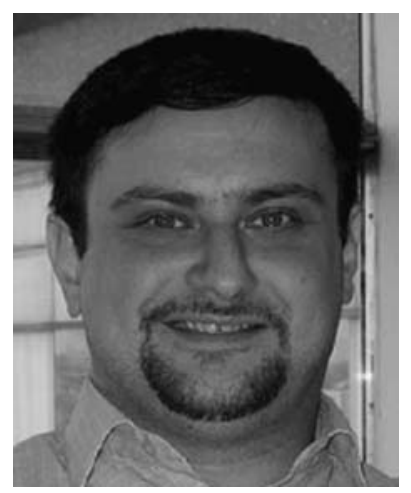

Dr. Nick Antonopoulos is currently a Senior Lecturer (US Associate Professor) at the Department of Computing, University of Surrey, UK. He holds a BSc in Physics (1st class) from the University of Athens in1993, an MSc in Information Technology from Aston University in 1994 and a PhD in Computer Science from the University of Surrey in 2000. He has published over 60 articles in fully refereed journals and international conferences. He has received a number of best paper awards in conferences and graduated four $\mathrm{PhD}$ students. He was the organiser and chair of the 1st international workshop on computational P2P networks (ComP2P 2008). He is currently the chair of the 1st international workshop on service-oriented P2P networks (ServP2P, CCGrid 2009) and the general chair of the international conference on advances in P2P systems (AP2PS 2009). He is on the editorial board of the Springer journal of Peer-to-Peer Networking and Applications (effective from 2009) and on the advisory editorial board of the IGI Global Handbook of Research on Telecommunications Planning and Management for Business. He is a Fellow of the UK Higher Education Academy and a full member of the British Computer Society. His research interests include emerging technologies such as large scale distributed systems and peer-to-peer networks, software agent architectures and security. Contact him at the Department of Computing, University of Surrey, Guildford, Surrey, GU2 7XH, United Kingdom; N.Antonopoulos@surrey.ac.uk 